Quim. Nova, Vol. 36, No. 6, 840-847, 2013

\title{
EFEITOS ENERGÉTICO-ESTRUTURAIS EM COMPOSTOS HETEROPOLICÍCLICOS COM OXIGÉNIO OU ENXOFRE
}

\author{
Vera L. S. Freitas e Maria D. M. C. Ribeiro da Silva* \\ Centro de Investigação em Química, Departamento de Química e Bioquímica, Faculdade de Ciências, Universidade do Porto, Rua \\ do Campo Alegre, 687, P-4169-007 Porto, Portugal \\ José R. B. Gomes \\ CICECO, Departamento de Química, Universidade de Aveiro, Campus Universitário de Santiago, P-3810-193 Aveiro, Portugal
}

Recebido em 30/10/12; aceito em 14/2/13; publicado na web em 15/4/13

\begin{abstract}
ENERGETIC AND STRUCTURAL EFFECTS ON OXYGEN OR SULFUR HETEROPOLYCYCLIC COMPOUNDS. The present article involves a comparative study of the influence of oxygen or sulfur heteroatoms present in the central ring of polycyclic compounds, in order to clarify the correlation between the respective thermophysical or thermochemical properties and structural characteristics. Considering the importance of these types of compounds for their broad spectrum of application in diverse fields, from pharmacology to the development of new materials, the critical interpretation of such properties for their crucial role in the reactivity of these substances is of great interest. Knowledge on these thermodynamic data for key compounds is also relevant to the prediction and understanding of the properties and behavior of other parent compounds.
\end{abstract}

Keywords: thermochemical and structural correlations; enthalpy of formation; heteropolycyclic compounds.

\section{INTRODUÇÃO}

\section{Energética e estrutura molecular - uma simbiose}

A disposição espacial dos átomos numa estrutura molecular, bem como a forma e a intensidade com que interagem, fazem parte da individualidade da molécula, determinando as respetivas caraterísticas energéticas e de reatividade. Para a avaliação, a compreensão e mesmo a previsão destas propriedades é essencial a fundamentação da estabilidade termodinâmica das moléculas, cujo suporte é sustentado pelo estudo de espécies "chave", representativas de diferentes classes de substâncias.

A Termoquímica Molecular tem um âmbito de interesses alargado, de que se destaca o estudo de propriedades termodinâmicas das moléculas, conducentes ao estabelecimento de relações energéticas das respetivas ligações químicas inter- e intramoleculares, bem como à sua correlação com as correspondentes propriedades estruturais e de reatividade. A presente revisão insere-se neste contexto, incidindo na quantificação e previsão das variações energéticas inerentes à presença de oxigénio ou enxofre num anel central (pentagonal ou hexagonal) integrante de estruturas policíclicas, com a avaliação complementar dos efeitos associados a diferentes grupos funcionais em diferentes posições.

O estudo termoquímico de diversas classes de compostos orgânicos, por recurso a estudos experimentais e computacionais, tem constituído um desafio motivador no Grupo de Energética Molecular, Colóides e Biointerfaces (CIQ-UP), no Departamento de Química e Bioquímica da Faculdade de Ciências da Universidade do Porto, não só pela sua contribuição para o conhecimento de parâmetros relevantes na interpretação da reatividade dos compostos, mas também por permitirem o estabelecimento de esquemas fiáveis de previsão das correspondentes propriedades para outras espécies estruturalmente afins.

Com o presente trabalho pretende-se contribuir para o esclarecimento da reatividade de alguns tipos de compostos heteropolicíclicos

*e-mail: mdsilva@fc.up.pt oxigenados ou sulfurados, estabelecendo correlações entre os respetivos parâmetros energéticos e estruturais.

\section{O contributo da Termoquímica}

Questionar o interesse de uma área científica é uma atitude frequente e saudável dos investigadores, talvez como uma consequência de, por vezes, a Ciência se manifestar como uma importante "linha de produção", em que a relevância e a visualização imediatas de "produto final concreto" são mais facilmente divulgadas e, talvez, de certa forma as mais valorizadas. Contudo, nenhuma Ciência evolui de forma isolada, necessitando de informação proveniente de outras áreas, que a suportem no seu desenvolvimento e ação. A própria história da Ciência evidencia que a evolução do conhecimento científico não é possível sem a contribuição e conjugação de várias áreas do saber, sendo o "individualismo científico" um sinónimo de retrocesso.

A aplicabilidade de parâmetros termoquímicos por si, ou conjugados com outros dados termofísicos relevantes para as substâncias consideradas é extensa e essencial ao desenvolvimento de estudos em diferentes domínios científicos. A título de exemplo, pode referir-se a importância da informação sobre a energia associada à formação e/ou transformação de moléculas em reações químicas, cujo conhecimento é fulcral na compreensão da sua estabilidade e reatividade, na previsão termoquímica de passos elementares de mecanismos reacionais e na definição de estratégias de otimização de processos químicos, onde tais moléculas participem. Em termos de aplicações mais recentes, é de evidenciar o recurso ao modelo QSAR (Quantitative Structure-Activity Relationship) ${ }^{1-3}$ no "design" de novos fármacos, onde o conhecimento da estrutura química, a par de determinadas propriedades físico-químicas são fundamentais para a identificação de compostos líderes e para o planeamento de análogos mais específicos, com uma atividade biológica superior (os efeitos eletrónicos inerentes a grupos substituintes influenciam, não raro, a atividade biológica de fármacos). O entendimento da natureza destes efeitos, bem como a sua quantificação, são de grande importância para a compreensão das relações entre a estrutura química e a atividade de compostos bioativos. Ainda na área da produção de fitofarmacêuticos, 
é de referir a importância do conhecimento da pressão de vapor das substâncias (e da sua dependência com a temperatura), propriedade física fundamental para o estabelecimento de períodos de toxicidade das mesmas. ${ }^{4}$

A importância dos estudos termoquímicos, em toda a sua extensão experimental e teórica, é evidenciada por uma descrição bastante peculiar, e muito atual, de Dudley R. Herschbach, laureado com o Prémio Nobel da Química em $1986,{ }^{5}$ pela sua contribuição nos estudos de dinâmica molecular de processos químicos elementares. No seu discurso de laureado, Herschbach refere a Termoquímica na base de uma grande montanha, que é necessário escalar para alcançar a Estrutura, seguida da Dinâmica e da Síntese posicionadas no seu topo, atingindo a Química Quântica, já situada nas nuvens, como último triunfo.

\section{Compostos heteropolicíclicos}

Os compostos policíclicos objeto deste estudo possuem dois anéis benzénicos fundidos a um anel central, pentagonal ou hexagonal, contendo um heteroátomo de oxigénio ou de enxofre, podendo esse anel conter também azoto (Figura 1).

A presença de átomos de enxofre ou de oxigénio numa estrutura molecular provoca, em geral, modificações a nível estrutural, com efeitos na energia e reatividade das espécies, que muitas vezes são fundamentais para o seu desempenho específico em diversas aplicações. O elemento representativo oxigénio e o elemento de transição interna enxofre pertencem ambos ao mesmo grupo na tabela periódica, apresentando idênticas configurações eletrónicas da camada de valência, o que se reflete em relações entre algumas propriedades dos compostos orgânicos contendo oxigénio na sua composição e as dos correspondentes compostos contendo enxofre. Contudo o átomo de enxofre, como elemento do terceiro período da Tabela Periódica, possui orbitais $d$ vazias que se encontram a uma curta distância energética o que permite a expansão da sua camada de valência e a consequente acomodação de até 10 ou 12 eletrões de valência. Nesta situação, o átomo de enxofre adquire uma estrutura de octeto expandido com hibridização $d s p^{3}$ ou $d^{2} s p^{3}$, respetivamente. Esta característica permite que os compostos de enxofre participem em reações inacessíveis aos correspondentes compostos de oxigénio.

A importância de compostos heteropolicíclicos em áreas diversas tem aumentando significativamente, através de muitos artigos publicados relativos à síntese, caracterização e desenvolvimento de novos derivados. A título de exemplo, citam-se algumas aplicações deste tipo de compostos: a indústria petrolífera tem um elevado interesse na remoção de enxofre presente na matéria orgânica fóssil, para reduzir o correspondente impacto ambiental e respeitar as normas de regulamentação do ar, ${ }^{6,7}$ pelo que o conhecimento das propriedades termoquímicas e termofísicas do dibenzotiofeno e seus derivados (compostos sulfurados mais comuns presentes no petróleo) são cruciais para que a sua remoção seja eficaz; o tioxanteno, o xanteno, a fenoxazina, a fenotiazina e seus derivados fazem parte dos esqueletos moleculares de inúmeros fármacos que apresentam múltiplas atividades biológicas e farmacológicas, ${ }^{8-22}$ sendo as suas propriedades físico-químicas relevantes para os métodos de QSAR; a tioxantona, a fenoxazina e a fenotiazina são utilizadas regularmente como substâncias fotoiniciadoras ${ }^{23-25}$ na indústria de polímeros; o xanteno ${ }^{26}$ e a fenoxazina ${ }^{27}$ fazem parte da estrutura de alguns corantes.

Atendendo à natureza dos heteroátomos, os compostos em estudo, cujas estruturas são apresentadas na Figura 1, foram divididos em compostos oxigenados (dibenzofurano, xanteno e fenoxazina) e sulfurados (dibenzotiofeno, tioxanteno e fenotiazina). Estas moléculas são as estruturas base deste estudo, a partir das quais todo o esquema de correlações se fundamenta. Em revistas científicas da especialidade ${ }^{28-35}$ encontram-se estudos termoquímicos para algumas destas moléculas, realizados por diferentes investigadores, cujos resultados apresentam uma gama de valores bastante dispersos para diferentes propriedades termoquímicas, dificultando a seleção/escolha do valor mais exato para cada composto (Tabela 1). Por esta razão, numa primeira fase, foram realizados novos estudos termoquímicos, complementados com estudos computacionais, empregando modernas aproximações de cálculo, numa tentativa de colmatar e/

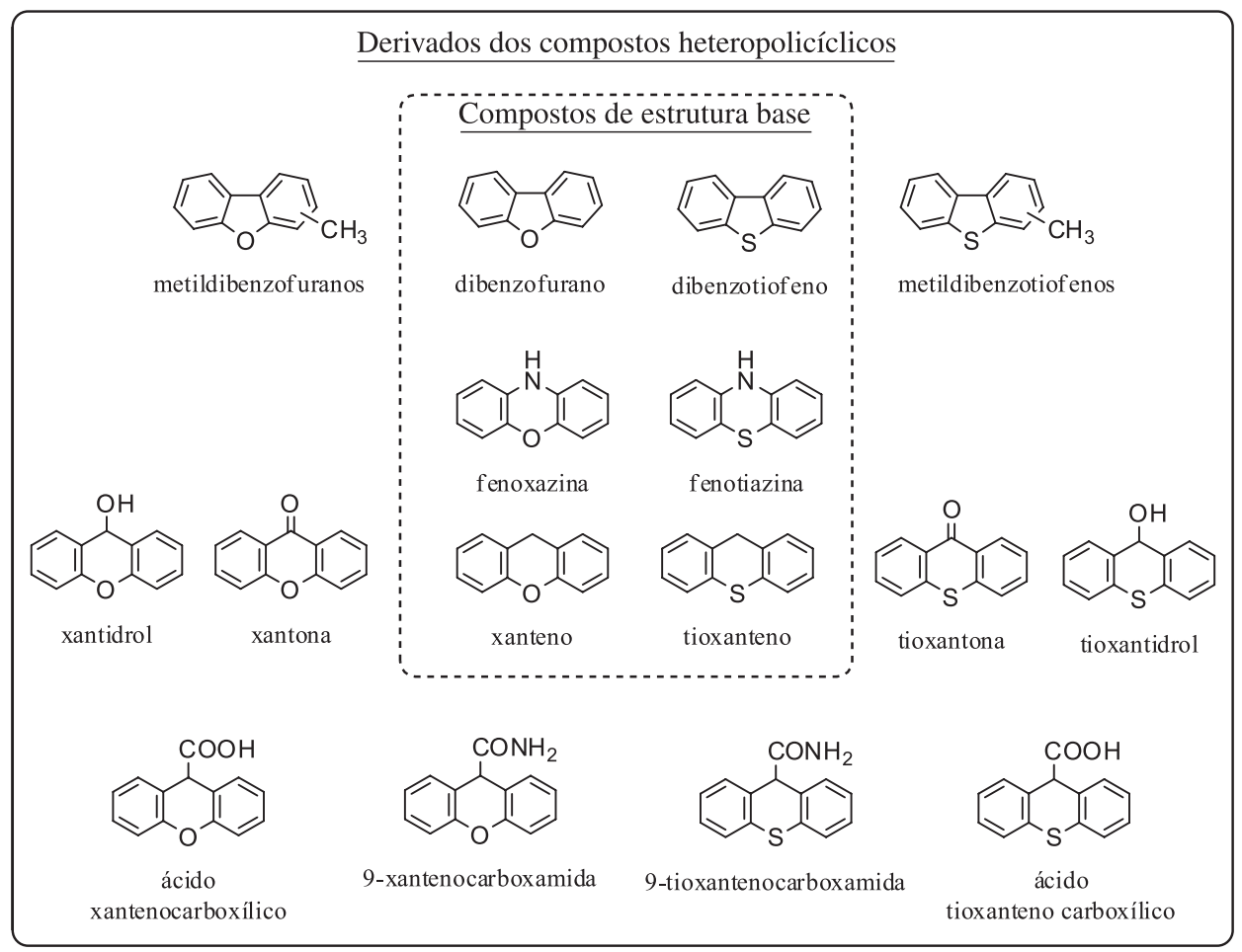

Figura 1. Estruturas moleculares dos compostos heteropolicíclicos de estrutura base dos respetivos derivados 
ou clarificar a situação, fornecendo, assim, valores que possam ser considerados fiáveis.

Numa fase posterior, foram estudados os compostos derivados destas estruturas base, como os derivados do dibenzofurano e do dibenzotiofeno com um substituinte metilo, bem como os derivados hidroxilo, cetona, ácido carboxílico e carboxamida do xanteno e do tioxanteno, apresentados na Figura 1.

Tabela 1. Valores termoquímicos disponíveis na literatura, anteriores a este estudo, para os compostos heteropolicíclicos e algumas generalidades

\begin{tabular}{|c|c|c|}
\hline Composto & $\begin{array}{c}\text { Valores da } \\
\text { literatura } \\
\Delta_{\mathrm{f}} H_{\mathrm{m}}^{\mathrm{o}}(\mathrm{g}) / \mathrm{kJ} \mathrm{mol}^{-1}\end{array}$ & Generalidades \\
\hline & $\begin{array}{c}(88.7 \pm 2.1)^{28} \\
(83.4 \pm 4.7)^{29} \\
(105.7 \pm 4.8)^{30} \\
(55.3 \pm 0.9)^{31} \\
(47.3 \pm 4.8)^{32}\end{array}$ & $\begin{array}{l}\text { O dibenzofurano é um composto cris- } \\
\text { talino de cor branca cujo processo de } \\
\text { purificação (fração mássica superior } \\
\text { a 0.999) é bastante difícil. }\end{array}$ \\
\hline & $(48.5 \pm 5.0)^{28,29,33}$ & $\begin{array}{l}\text { Os derivados do xanteno mais conhe- } \\
\text { cidos e utilizados em diversas áreas } \\
\text { são os que possuem o grupo cetona } \\
\text { em para posição relativamente ao } \\
\text { heteroátomo de oxigénio. }\end{array}$ \\
\hline & $(94.1 \pm 2.8)^{34}$ & $\begin{array}{l}\text { A fenoxazina apresenta-se sob a } \\
\text { forma de cristais amarelos. Muitos } \\
\text { dos seus derivados são utilizados } \\
\text { como corantes na indústria têxtil. }\end{array}$ \\
\hline & $\begin{array}{l}(189.3 \pm 4.5)^{30} \\
(213.8 \pm 0.8)^{35} \\
(205.1 \pm 1.5)^{29}\end{array}$ & $\begin{array}{l}\text { Para o dibenzotiofeno existe um } \\
\text { elevado número de estudos de tran- } \\
\text { sição de fase. }\end{array}$ \\
\hline & $(278.2 \pm 1.9)^{29,34}$ & $\begin{array}{l}\text { Os neurolépticos tricíclicos caracter- } \\
\text { izam-se por possuírem um anel de } \\
\text { fenotiazina ou um anel de tioxanteno. }\end{array}$ \\
\hline
\end{tabular}

\section{PARTE EXPERIMENTAL}

\section{Entalpia molar de formação padrão no estado gasoso -} determinação por via experimental

As interações intermoleculares de um composto, no estado gasoso, a baixa pressão, podem ser consideradas desprezáveis. Por esta razão, o estado gasoso é considerado como referência para o estabelecimento de relações entre a estrutura, a energia e a reatividade (Figura 2). A partir do conhecimento dos valores da entalpia molar de formação padrão de diferentes compostos, no estado gasoso, $\Delta_{\mathrm{f}} H_{\mathrm{m}}^{\mathrm{o}}(\mathrm{g})$, é, assim, possível avaliar os efeitos entálpicos provocados pela presença de grupos substituintes distintos, comparando-os com

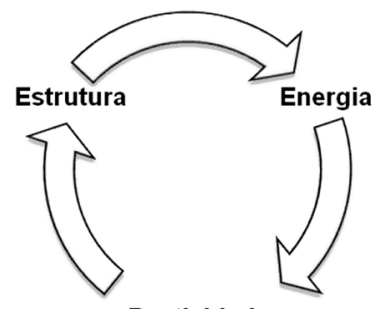

Reatividade

Figura 2. Esquema representativo das relações estrutura/energia/reatividade, em fase gasosa os de moléculas estruturalmente semelhantes, com o objetivo final de adquirir informação suficiente para estabelecer esquemas de previsão de propriedades relativas a compostos cujo estudo experimental não seja possível.

A determinação experimental direta da entalpia molar de formação padrão, no estado gasoso, raramente é viável, pelo que um procedimento alternativo passa pela conjugação de parâmetros energéticos em fase condensada com outros referentes a transições de fase, todos determinados a partir de medições experimentais. De facto, para um dado composto orgânico, a medição das suas propriedades energéticas em fase condensada (cristalina ou líquida), possibilita a determinação do valor da respetiva entalpia molar de formação padrão, $\Delta_{\mathrm{f}} H_{\mathrm{m}}^{\mathrm{o}}(\mathrm{cr}, 1)$, em que são considerados os efeitos energéticos das interações intra- e intermoleculares. Para se calcular a entalpia de formação do mesmo composto no estado gasoso, a partir da entalpia de formação no estado condensado, é necessário considerar o efeito energético das forças intermoleculares, recorrendo à determinação de entalpias de transição de fase, $\Delta_{\mathrm{cr}, 1}^{\mathrm{g}} H_{\mathrm{m}}^{\mathrm{o}}$. Neste trabalho a calorimetria de combustão e a microcalorimetria Calvet ou o método de efusão de Knudsen foram as técnicas experimentais utilizadas para determinar os parâmetros termoquímicos anteriormente citados, respetivamente (Figura 3).

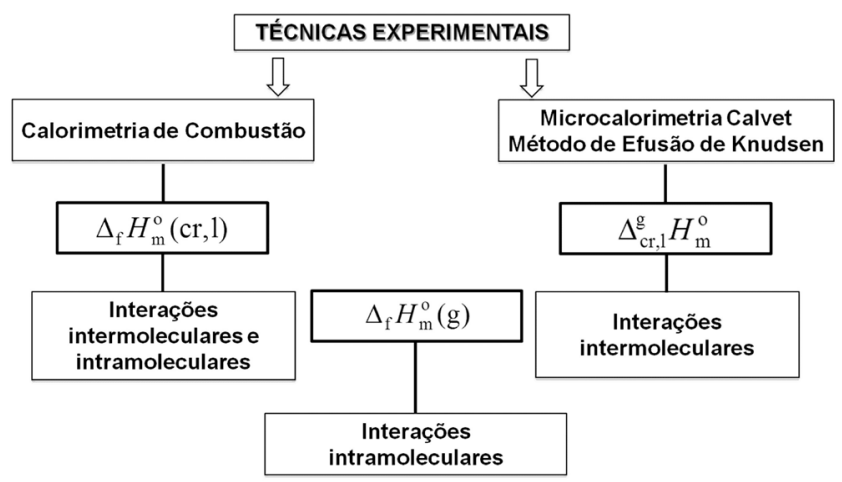

Figura 3. Diagrama representativo das propriedades obtidas por via experimental

\section{A calorimetria de combustão no estudo energético de fases condensadas}

A calorimetria de combustão é uma das técnicas físico-químicas mais antigas, surgida na segunda metade do século XIX, introduzida por Berthelot, ${ }^{36}$ para a obtenção de vários parâmetros termodinâmicos, nomeadamente, entalpias de formação de compostos orgânicos de baixa reatividade, sólidos ou líquidos. Os princípios basilares desta técnica mantiveram-se inalterados, consistindo na medição da quantidade de energia (a volume constante) desenvolvida numa reação química em que ocorre a oxidação completa de uma substância, sendo as respectivas ligações químicas completamente rompidas. A reação ocorre num vaso reacional (bomba de combustão) cheio de oxigénio sob pressão (cerca de $3.04 \mathrm{MPa}$ ) e em condições experimentais bem definidas, devendo ser rápida e completa, sem a formação de produtos secundários e além da necessidade elevada pureza do composto a queimar, as espécies químicas presentes nos estados inicial e final devem ser facilmente caracterizáveis. O calor proveniente da reação é calculado a partir da medição da variação de temperatura do líquido calorimétrico (água ou outro fluído com elevada condutividade térmica) que envolve o vaso reacional.

O valor da energia de combustão (energia interna) é derivado por comparação das variações de temperaturas, rigorosamente determinadas, em ensaios paralelos de combustão de um padrão termoquímico e 
do composto em estudo. Este valor deve ser posteriormente corrigido para o estado padrão $\left(p^{\circ}=0.1 \mathrm{MPa}\right)$ - correções de Washburn ${ }^{37}$ - e para uma temperatura de referência $(T=298.15 \mathrm{~K})$.

Conhecendo a variação de energia interna, $\Delta_{\mathrm{c}} U_{\mathrm{m}}^{\circ}$, e a variação da quantidade de substância, em fase gasosa, $\Delta n$, envolvida na reação de combustão, é possível calcular a entalpia molar de combustão padrão, $\Delta_{\mathrm{c}} H_{\mathrm{m}}^{\mathrm{o}}$, à temperatura considerada, a partir da relação, $\Delta_{\mathrm{c}} H_{\mathrm{m}}^{\circ}=\Delta_{\mathrm{c}} U_{\mathrm{m}}^{\mathrm{o}}+\Delta n R T$, onde $R$ é a constante dos gases e $T$ a temperatura de referência $(298.15 \mathrm{~K})$.

Neste género de determinações calorimétricas, o calorímetro usado é do tipo isoperibol, sendo a temperatura da vizinhança mantida constante e uniforme, qualquer que seja a temperatura no vaso calorimétrico. A vizinhança possui uma capacidade calorífica elevada e a resistência térmica entre esta e o calorímetro, onde ocorre o processo químico em estudo, é bem definida. Assim, é permitida a transferência de calor entre o sistema e a vizinhança e, como estas trocas de calor são devidamente controladas, facilmente se conhece a quantidade de calor transferido.

A composição qualitativa dos compostos submetidos a estudo termoquímico condiciona a seleção do método calorimétrico a utilizar, assim, para compostos orgânicos que possuam na sua composição apenas $\mathrm{C}, \mathrm{H}, \mathrm{O}$ e N é utilizada a calorimetria de combustão em bomba estática, enquanto que para compostos contendo, além dos elementos referidos, halogéneos, fósforo e enxofre, é utilizada a calorimetria de combustão em bomba rotativa. A técnica de calorimetria de combustão em bomba rotativa também tem sido utilizada com sucesso no estudo de alguns compostos organometálicos. Os sistemas calorimétricos de combustão, usados no estudo dos compostos analisados no presente trabalho, estão pormenorizadamente descritos na literatura (bomba estática ${ }^{38-40}$ para compostos contendo oxigénio e bomba rotativa ${ }^{41}$ para compostos contendo enxofre; em anexo são apresentadas imagens destes sistemas).

\section{Técnicas de calorimetria e de efusão no estudo de transições de fase}

A entalpia molar de transição de uma fase condensada para a gasosa permite uma estimativa da ordem de grandeza das forças intermoleculares. Este parâmetro pode ser determinado experimentalmente a partir de um método direto, em que a energia necessária para sublimar ou vaporizar, isotermicamente, uma quantidade de composto rigorosamente conhecida, é medida num calorímetro, ou por um método indireto em que é efetuada a medição de uma propriedade, como a pressão de vapor do composto a diferentes temperaturas, que permite o cálculo da entalpia molar de transição de fase, através da equação de Clausius-Clapeyron.

A técnica de microcalorimetria Calvet permite uma medição direta das entalpias de sublimação e vaporização. $\mathrm{O}$ funcionamento atual do microcalorímetro Calvet é baseado no modelo do calorímetro de células gémeas desenvolvido por Calvet, em 1948. ${ }^{42,43}$ Essencialmente, este microcalorímetro é constituído por duas células calorimétricas idênticas (célula da amostra onde ocorre o fenómeno térmico e célula de referência que permite compensar os efeitos irregulares de calor ${ }^{44}$ ) dispostas simetricamente em duas cavidades, num bloco metálico de elevada capacidade calorífica, controlado isotermicamente, com grande precisão, para uma dada temperatura pré-selecionada $T$. Numa determinação experimental, são lançados, à temperatura ambiente, em simultâneo dois capilares de vidro, de massas muito próximas nas respetivas células calorimétricas; quando os capilares atingem a termoestabilidade, a amostra é vaporizada/ sublimada e removida por vácuo da zona quente do calorímetro. $\mathrm{O}$ estudo termocinético do processo é obtido a partir da medição do fluxo de calor com o tempo, definindo-se um termograma, cuja área, após tratamento adequado, permite calcular a variação entálpica total medida para o processo de transição de fase.

No âmbito do estudo experimental aqui desenvolvido para a sublimação de sólidos e vaporização de líquidos foi utilizada a mesma técnica de microcalorimetria em alto-vácuo descrita por Skinner e co-autores, ${ }^{45}$ para a sublimação de sólidos. A medição de entalpias de vaporização utilizando esta técnica foi iniciada por Ribeiro da Silva e colaboradores, com resultados bastante satisfatórios. ${ }^{46} \mathrm{Na}$ literatura ${ }^{47}$ é possível consultar com pormenor o sistema de microcalorimetria Calvet instalado no nosso laboratório (em anexo são apresentadas imagens desse sistema).

O método de efusão de Knudsen surgiu em 1909 com Martin Knudsen, ${ }^{48-51}$ com a introdução da célula de efusão. Em linhas gerais, este método consiste na determinação da pressão de vapor a partir da variação de massa de um composto na célula de efusão, provocada pelo fluxo do composto no estado de vapor, através de um orifício de dimensão reduzida, existente na tampa da célula, durante um determinado tempo de efusão e a uma dada temperatura. Este tipo de método requer medições cuidadosas, pois os erros experimentais associados às determinações de pressão e temperatura, podem ser ampliados no processo de cálculo da entalpia de sublimação, a partir do declive da função $\ln p=\mathrm{f}(1 / T)$.

A equação utilizada para a determinação de entalpias de sublimação é a forma integrada da equação de Clausius-Clapeyron.

O sistema de efusão de Knudsen usado no estudo dos compostos aqui apresentados foi construído e testado no nosso departamento ${ }^{52}$ (em anexo, apresenta-se uma imagem do sistema), tendo o seu bom funcionamento em termos de exatidão e precisão sido confirmado medindo as pressões de vapor, entre 0.1 e $1 \mathrm{~Pa}$, para intervalos de temperaturas de $20 \mathrm{~K}$, dos seguintes compostos: ácido benzóico, fenantreno, antraceno, benzantreno e 1,3,5-trifenilbenzeno. Este sistema permite a operação simultânea de nove células de efusão, dispostas em três fornos com temperaturas independentes, cada um dos quais contém três células com orifícios de efusão de diferente tamanho, mantidas a uma dada temperatura controlável para cada um dos fornos. Assim, num único ensaio experimental, são determinadas as pressões de vapor da substância para três temperaturas diferentes.

\section{Entalpia molar de formação padrão no estado gasoso - determinação por via computacional}

Outro modo de calcular as entalpias de formação no estado gasoso passa pela estimativa dos valores utilizando métodos modernos de cálculo. Recentemente, o enorme desenvolvimento das aproximações de Química Computacional, aliado à constante evolução dos sistemas informáticos (processadores, memórias, discos, etc. mais rápidos), tem permitido a realização de cálculos para sistemas químicos mais complexos e/ou de maiores dimensões, e consequentemente, tem contribuído de forma significativa para um conhecimento mais alargado de parâmetros termoquímicos relativos a um grande número de compostos. No entanto, os métodos usados nesta área, denominada de Termoquímica Computacional, são dependentes do conhecimento de valores experimentais fiáveis para as suas bases de cálculo e muitas vezes cruciais na estimativa de valores teóricos de outros compostos. As determinações experimentais têm, assim, uma inegável importância, no estabelecimento de outros métodos de previsão de parâmetros, podendo ainda referir-se a complementaridade e validação recíproca dos valores obtidos pela via experimental e por cálculo.

Nos estudos computacionais aqui reportados, a energia das moléculas foi determinada pelo método composto G3(MP2)//B3LYP. ${ }^{53}$ Neste método a otimização da geometria é feita com recurso à teoria dos funcionais da densidade, DFT, usando o método B3LYP, ${ }^{54,55} \mathrm{com}$ a base 6-31G $(d)$. Para uma melhor compreensão destas técnicas 
de cálculo, o leitor poderá estar interessado em consultar um livro especializado nesta área. ${ }^{56} \mathrm{~A}$ energia do ponto zero é obtida ao mesmo nível téorico (B3LYP/6-31G $(d)$ ) e as frequências de vibração são multiplicadas por um fator de escala $(0.9613)^{57}$ para corrigir imperfeições do método que as sobrestimam. A energia da molécula obtida ao nível B3LYP/6-31G $(d)$, cálculo de qualidade modesta, é depois corrigida através de dois cálculos pontuais adicionais mais completos - métodos QCISD $(\mathrm{T})^{58}$ e MP2 $2^{59}$ - o último dos quais com uma base bastante alargada, introduzindo-se, assim, os efeitos da correlação eletrónica de todos os eletrões com o primeiro destes cálculos e corrigindo-se o efeito do tamanho da base no segundo dos cálculos pontuais. Com base no conhecimento do valor da energia de moléculas é possível estimar a entalpia molar de formação padrão para um dado composto, a partir de reações de substituição de grupos (isodésmicas, homodesmóticas, etc.) ${ }^{60-63}$ e sabendo os valores experimentais das entalpias de formação para todas as outras espécies intervenientes nessas reações.

\section{RESULTADOS E DISCUSSÃO}

Os parâmetros termoquímicos obtidos para os compostos heteropolicíclicos, por via experimental, $\left(\Delta_{\mathrm{f}} H_{\mathrm{m}}^{\mathrm{o}}(\mathrm{cr}), \Delta_{\mathrm{cr}}^{\mathrm{g}} H_{\mathrm{m}}^{\mathrm{o}}\right.$ e $\left.\Delta_{\mathrm{f}} H_{\mathrm{m}}^{\circ}(\mathrm{g})^{\exp }\right)$, e por via computacional $\left(\Delta_{\mathrm{f}} H_{\mathrm{m}}^{\circ}(\mathrm{g})^{\text {comp }}\right)$ são apresentados na Tabela 2. Nas referências ${ }^{64-73}$ assinaladas junto dos valores é possível consultar com mais detalhe os estudos experimentais e computacionais desenvolvidos.

No conjunto de compostos apresentados, o número de compostos oxigenados disponíveis é superior ao dos compostos sulfurados afins,

Tabela 2. Valores das entalpias molares de formação padrão, no estado condensado e gasoso, e das entalpias molares de transição da fase condensada para a gasosa, para os compostos estudados. Valores em $\mathrm{kJ} \cdot \mathrm{mol}^{-1}$

\begin{tabular}{|c|c|c|c|c|c|}
\hline Compostos Oxigenados & $\begin{array}{c}\Delta_{\mathrm{f}} H_{\mathrm{m}}^{\mathrm{o}}(\mathrm{cr}) \\
\Delta_{\mathrm{cr}}^{\mathrm{g}} H_{\mathrm{m}}^{\mathrm{o}}\end{array}$ & $\begin{array}{c}\Delta_{\mathrm{f}} H_{\mathrm{m}}^{\mathrm{o}}(\mathrm{g})^{\exp } \\
\Delta_{\mathrm{f}} H_{\mathrm{m}}^{\mathrm{o}}(\mathrm{g})^{\operatorname{comp}} \\
\end{array}$ & Compostos Sulfurados & $\begin{array}{c}\Delta_{\mathrm{f}} H_{\mathrm{m}}^{\mathrm{o}}(\mathrm{cr}) \\
\Delta_{\mathrm{cr}}^{\mathrm{g}} H_{\mathrm{m}}^{\mathrm{o}}\end{array}$ & $\begin{array}{r}\Delta_{\mathrm{f}} H_{\mathrm{m}}^{\mathrm{o}}(\mathrm{g})^{\exp } \\
\Delta_{\mathrm{f}} H_{\mathrm{m}}^{\mathrm{o}}(\mathrm{g})^{\mathrm{comp}}\end{array}$ \\
\hline Dibenzofurano (cr) & $\begin{array}{l}(-29.2 \pm 3.8)^{64} \\
(84.5 \pm 1.0)^{64}\end{array}$ & $\begin{array}{l}(55.0 \pm 3.9)^{64} \\
(55.0 \pm 0.4)^{64}\end{array}$ & Dibenzotiofeno (cr) & $\begin{array}{c}(118.1 \pm 4.5)^{65} \\
(93.2 \pm 0.5)^{65}\end{array}$ & $\begin{array}{c}(211.3 \pm 4.5)^{65} \\
(212.2 \pm 0.8)^{64,65}\end{array}$ \\
\hline Xante & $\begin{array}{c}(-50.2 \pm 3.3)^{66} \\
(92.0 \pm 1.1)^{66}\end{array}$ & $\begin{array}{l}(41.8 \pm 3.5)^{66} \\
(41.9 \pm 0.7)^{67}\end{array}$ & Tioxante & $\begin{array}{l}(117.4 \pm 4.1)^{68} \\
(101.3 \pm 0.8)^{68}\end{array}$ & $\begin{array}{l}(218.7 \pm 4.2)^{68} \\
(220.1 \pm 0.9)^{67}\end{array}$ \\
\hline Fenoxazir & $\begin{array}{l}(-1.6 \pm 4.1)^{64} \\
(102.4 \pm 1.7)^{64}\end{array}$ & $\begin{array}{l}(100.8 \pm 4.4)^{64} \\
(101.8 \pm 0.9)^{64}\end{array}$ & Fenotiazina (cr) & $\begin{array}{l}(162.5 \pm 4.4)^{64} \\
(103.3 \pm 0.9)^{64}\end{array}$ & $\begin{array}{l}(265.8 \pm 4.5)^{64} \\
(264.3 \pm 0.6)^{64}\end{array}$ \\
\hline 1-Metildibenzofurano & - & $\frac{-}{(18.0 \pm 0.4)^{64}}$ & 1-Metildibenzotiofeno & - & $\frac{-}{(182.2 \pm 0.5)^{69}}$ \\
\hline 2-Metildibenzofurano & - & $(24.5 \pm 0.3)^{64}$ & 2-Metildibenzotiofeno & - & $(180 . \overline{1} \pm 0.5)^{69}$ \\
\hline 3-Metildibenzofurano & 一 & $(23.4 \pm 0.3)^{64}$ & 3-Metildibenzotiofeno & - & $(179 . \overline{-} \pm 0.4)^{69}$ \\
\hline 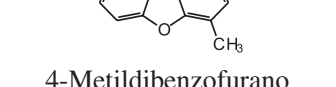 & - & $(18.1 \pm 0.4)^{64}$ & 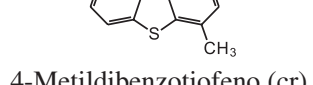 & $\begin{array}{l}(70.9 \pm 4.8)^{70} \\
(90.3 \pm 1.4)^{70}\end{array}$ & $\begin{array}{l}(161.2 \pm 4.9)^{70} \\
(172.5 \pm 0.5)^{69}\end{array}$ \\
\hline & $\begin{array}{c}(-196.7 \pm 4.0)^{71} \\
(102.7 \pm 2.3)^{71}\end{array}$ & $\begin{array}{l}(-94.0 \pm 4.6)^{71} \\
(-97.3 \pm 1.0)^{67}\end{array}$ & & $\begin{array}{l}(-14.6 \pm 2.1)^{72} \\
(106.5 \pm 1.2)^{72}\end{array}$ & $\begin{array}{l}(91.9 \pm 2.4)^{72} \\
(93.1 \pm 1.2)^{67}\end{array}$ \\
\hline & $\begin{array}{c}(-236.4 \pm 4.3)^{73} \\
(115.2 \pm 1.7)^{73}\end{array}$ & $\begin{array}{l}(-121.2 \pm 4.6)^{73} \\
(-124.0 \pm 0.9)^{73}\end{array}$ & & - & $(50.5 \pm 0.8)^{67}$ \\
\hline Ácido xan & $\begin{array}{c}(-441.3 \pm 2.8)^{73} \\
(128.2 \pm 3.7)^{73}\end{array}$ & $\begin{array}{l}(-313.1 \pm 4.6)^{73} \\
(-317.5 \pm 2.1)^{73}\end{array}$ & $\begin{array}{l}\text { Ácido tioxanteno-9- } \\
\text { carboxílico }\end{array}$ & - & $(-144.9 \pm 0.4)^{67}$ \\
\hline & $\begin{array}{c}(-270.8 \pm 2.1)^{73} \\
(130.0 \pm 4.2)^{73}\end{array}$ & $\begin{array}{l}(-140.8 \pm 4.7)^{73} \\
(-142.8 \pm 0.9)^{73}\end{array}$ & & - & $(30.7 \pm 0.9)^{67}$ \\
\hline Xanteno-9-carboxamida (cr) & & & Tioxanteno-9-carboxamida & & \\
\hline
\end{tabular}


devido à escassa oferta comercial existente e, em alguns casos, a quantidade de impurezas ser muito elevada, tornando impossível obter quantidades suficientes de amostras de elevada pureza necessárias para estudo termoquímico experimental.

Assim, uma das estratégias deste trabalho foi estudar, paralelamente, em nível experimental e computacional o maior número possível de compostos oxigenados e alguns correspondentes compostos sulfurados. A boa concordância entre os valores de $\Delta_{\mathrm{f}} H_{\mathrm{m}}^{\mathrm{o}}(\mathrm{g})^{\exp }$ e $\Delta_{\mathrm{f}} H_{\mathrm{m}}^{\mathrm{o}}(\mathrm{g})^{\text {comp }}$ permitiu testar e validar ${ }^{67}$ a aplicabilidade do método computacional a este tipo de compostos e, ainda estimar a entalpia molar de formação padrão para outros compostos que não foram estudados experimentalmente, pela não disponibilidade dos mesmos em pureza elevada e em quantidades suficientes para os estudos termoquímicos experimentais.

Na tentativa de compreender e prever o comportamento termoquímico do conjunto de compostos apresentado (Figura 1), verificou-se que os compostos heterocíclicos com um anel central de seis lados e os de cinco lados se relacionam de forma diferente, sendo por isso apresentadas duas correlações.

Na primeira correlação, utilizando os resultados obtidos para os compostos contendo um anel central de seis lados e com substituintes em posição para relativamente ao heteroátomo foi elaborada uma representação gráfica (Figura 4) entre a entalpia molar de formação padrão, no estado gasoso dos compostos sulfurados e dos compostos oxigenados, a partir da qual foi possível obter uma ótima relação linear com um coeficiente de correlação, $R^{2}=0.9975$, calculada por regressão linear.

Para os restantes compostos, contendo um anel central de cinco lados, foi realizado o mesmo tipo de representação gráfica, entre a entalpia molar de formação padrão, no estado gasoso, dos compostos sulfurados e dos compostos oxigenados, obtendo-se uma relação linear fraca. Da análise dos resultados verificou-se que os valores que se afastavam de uma possível relação linear, correspondiam aos compostos com substituintes metilo nas posições 1 e 4 . Retirando estes valores e acrescentando os outros disponíveis na literatura para outros compostos cíclicos contendo heteroátomos de oxigénio ou enxofre, como o furano $(-34.8 \pm 0.7) \mathrm{kJ} \mathrm{mol}^{-1},{ }^{29} \mathrm{o}$ tiofeno $(115.0 \pm$ 1.0) $\mathrm{kJ} \mathrm{mol}^{-1},{ }^{29}$ o benzofurano $(13.6 \pm 0.7) \mathrm{kJ} \mathrm{mol}^{-1,74} \mathrm{e}$ o benzotiofeno $(166.3 \pm 0.9) \mathrm{kJ} \mathrm{mol}^{-1},{ }^{29}$ foi possível construir uma nova representação gráfica (Figura 5), com uma relação linear bastante satisfatória, sendo o seu valor de $R^{2}$ igual a 0.9990 .

Estas relações podem ser usadas para estimar os valores das entalpias molares de formação padrão dos compostos heteropolicíclicos,

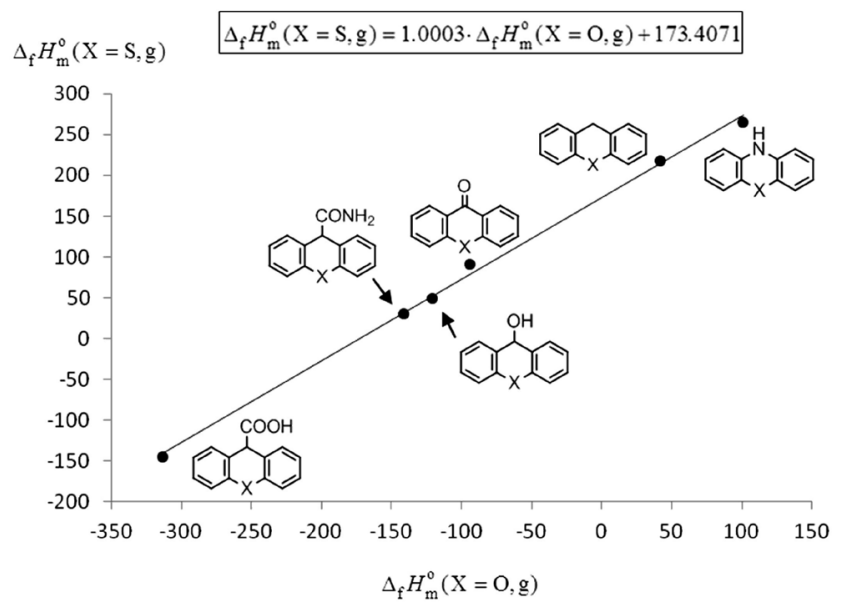

Figura 4. Correlação entre as entalpias molares de formação padrão, no estado gasoso, para compostos com um anel central hexagonal com heteroátomos de enxofre $(X=S)$ ou oxigénio $(X=O)$. Valores em $\mathrm{kJ} \cdot \mathrm{mol}^{-1}$

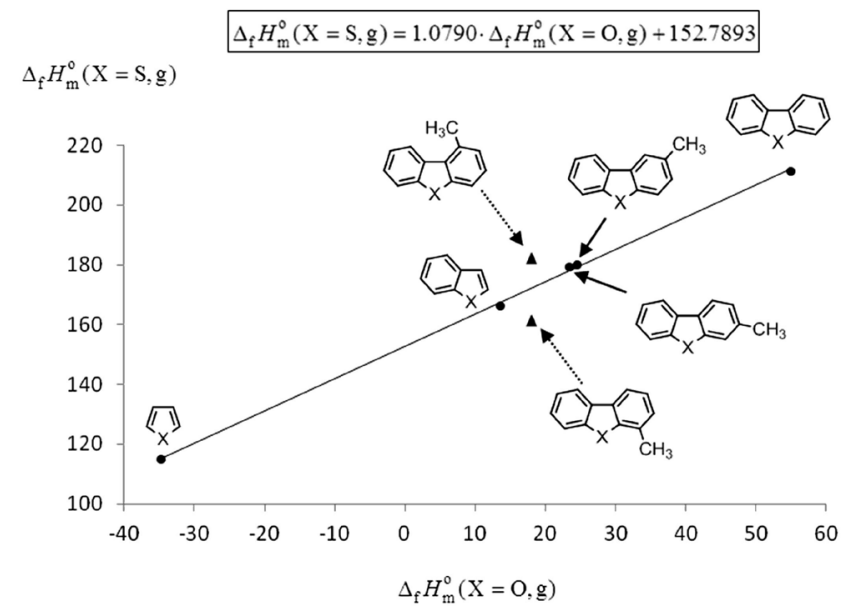

Figura 5. Correlação entre as entalpias molares de formação padrão, no estado gasoso, para compostos contendo um anel pentagonal com heteroátomos de enxofre $(X=S)$ ou oxigénio $(X=O)$. Código de símbolos: •, compostos utilizados no estabelecimento da correlação; $\mathbf{\Delta}$, compostos manifestando comportamento desviante. Valores em $\mathrm{kJ} \cdot \mathrm{mol}^{-1}$

contendo oxigénio ou enxofre, com algumas limitações, como a posição dos substituintes.

\section{CONCLUSÃO}

No contexto deste estudo, o trabalho experimental e computacional desenvolvido permitiu a caracterização estrutural e termofísicoquímica de um conjunto de compostos heteropolicíclicos, alguns deles compostos "chave" para a avaliação comparativa e interpretação de efeitos energéticos causados pela substituição de um átomo de oxigénio por um átomo enxofre.

A termoquímica computacional complementou a informação obtida experimentalmente, e provou ser uma alternativa à parte experimental, desde que os resultados teóricos sejam calibrados previamente para moléculas semelhantes.

$\mathrm{O}$ desenvolvimento deste estudo contribuiu significativamente para o alargamento da escassa base de parâmetros termoquímicos disponível para esta família de compostos heteropolicíclicos, com grande importância do ponto de vista prático, pela possibilidade de serem utilizados nos mais diversos contextos, associados às inúmeras aplicações destes compostos.

Futuramente, será bastante interessante desenvolver este estudo termoquímico para derivados com outros grupos funcionais em diferentes posições estruturais dos anéis, disponibilizando-se para esta família de compostos uma base de dados termoquímicos mais alargada que consolide o estabelecimento de um método de grupos, sólido e fiável, em que as possíveis interações entre grupos sejam consideradas, proporcionando estimativas, de elevado grau de confiança, para valores de entalpias de formação de difícil ou inviável determinação experimental.

\section{MATERIAL SUPLEMENTAR}

Algumas imagens dos sistemas utilizados neste trabalho estão disponíveis em http://quimicanova.sbq.org.br, na forma de arquivo PDF, com acesso livre.

\section{AGRADECIMENTOS}

À Fundação para a Ciência e Tecnologia e ao Fundo Social Europeu, no âmbito do Quadro Comunitário de Apoio. V. L. 
S. F. agradece a concessão de uma bolsa de Doutoramento (BD/41672/2007) e o apoio financeiro que permitiu a divulgação de parte deste trabalho em várias conferências científicas internacionais da especialidade, bem como a concessão de uma bolsa de Pósdoutoramento (BPD/78552/2011). Outro agradecimento é devido ao Centro de Investigação em Química da Universidade do Porto, CIQUP (Pest-C/QUI/UI0081/2011), pela disponibilização das condições necessárias à concretização deste trabalho.

\section{REFERÊNCIAS}

1. Tavares, L. C.; Quim. Nova 2004, 27, 631.

2. Gaudio, A. C.; Zandonade, E.; Quim. Nova 2001, 24, 658.

3. Arroio, A; Honório K. M.; da Silva, A. B. F.; Quim. Nova 2010, 33, 694.

4. Ferguson J.; Proc. R. Soc. Lond. B. Biol. Sci. 1939, 127, 387.

5. Dudley R. Herschbach - Nobel Lecture; http://nobelprize.org/ nobel_prizes/chemistry/laureates/1986/herschbach-lecture.html, acessada em Outubro 2012.

6. http://ec.europa.eu/environment/air/legis.htm, acessada em Outubro 2012.

7. www.epa.gov/tier2/amendments/420f06027.htm, acessada em Outubro 2012.

8. Julien, R. M.; A Primer of Drug Action, $5^{\text {th }}$ ed., W. H. Freeman and Company: Portland, OR, 1988.

9. Liska, K.; Drug and the Human Body, $6^{\text {th }}$ ed., Prentice Hall: San Diego, CA, 1999.

10. Belal, F.; Hefnawy, M. M.; Aly, F. A.; J. Pharm. Biomed. Anal. 1997, $16,369$.

11. Harder, A.; Parasitol. Res. 2002, 88, 395.

12. Palmeira, A.; Vasconcelos, M. H.; Paiva, A.; Fernandes, M. X.; Pinto, M.; Sousa, E.; Biochem. Pharmacol. 2012, 83, 57.

13. Corbett, T. H.; Panchapor, C.; Polin, L.; Lowichik, N.; Pugh, S.; White, K.; Kushner, J.; Meyer, J.; Czarnecki, J.; Chinnukroh, S.; Invest. New Drugs 1999, 17, 17.

14. Stevenson, J. P.; De Maria, D.; Reilly, D.; Purvis, J. D.; Graham, M. A.; Cancer Chemoter. Pharmacol. 1999, 44, 228.

15. Woo, S.; Jung, J; Lee, C.; Kwon, Y.; Na, Y.; Bioorg. Med. Chem. Lett. 2007, 17, 1163.

16. Castanheiro, R. A. P.; Pinto, M. M. M.; Silva, A. M. S.; Cravo, S. M. M.; Gales, L.; Damas, A. M.; Bioorg. Med. Chem. 2007, 15, 6080.

17. Pinto, M. M. M.; Sousa, M. E.; Nascimento, M. S. J.; Curr. Med. Chem. 2005, 12, 2517.

18. Dharmaratne, H. R. W.; Wijesinghe, W. M. N. M.; Thevanasem, V.; J. Ethnopharmacol. 1999, 66, 339.

19. Kumar, R.; Kumari, M.; J. Chem. Pharm. Res. 2011, 3, 217.

20. Korth, C.; May, B. C. H.; Cohen, F. E.; Prusiner, S. B.; PNAS 2001, 98, 9836.

21. Naya, A.; Sagara, Y.; Ohwaki, K.; Saeki, T.; Ichikawa, D.; Iwasawa, Y.; Noguchi, K.; Ohtake, N.; J. Med. Chem. 2001, 44, 1429.

22. Jastrzębska-Więsek, M.; Librowski, T.; Czarnecki, R.; Marona, H.; Nowak, G.; Pol. J. Pharmacol. 2003, 55, 461.

23. Lalevée, J.; Blanchard, N.; Tehfe, M. A.; Fries, C.; Morlet-Savary, F.; Gigmes, D.; Fouassier, J. P.; Polym. Chem. 2011, 2, 1077.

24. Villegas, L.; Encinas, M. V.; Rufs, A. M.; Bueno, C.; Bertolotti, S.; Previtali, C. M.; J. Polym. Sci., Part A: Polym. Chem. 2001, 39, 4074.

25. Gomurashvili, Z.; Crivello, J. V.; J. Polym. Sci., Part A: Polym. Chem. 2001, 39, 1187.

26. Fleming, G. R.; Knight, A. W. E.; Morris, J. M.; Morrison, R. J. S.; Robinson G. W.; J. Am. Chem. Soc. 1977, 99 , 4306.

27. Jose, J.; Burgess. K.; Tetrahedron 2006, 62, 11021.

28. Cass, R. C.; Fletcher, S. E.; Mortimer, C. T.; Springall, H. D.; White, T. R.; J. Chem. Soc. 1958, 1406.

29. Pedley, J. B.; Thermochemical Data and Structures of Organic Com- pounds, Thermodynamics Research Center: College Station, TX, 1994. 30. Sabbah, R.; Antipine I.; Bull. Soc. Chim. Fr. 1987, 124, 392.

31. Chirico, R. D.; Gammon, B. E.; Knipmeyer, S. E.; Nguyen, A.; Strube, M. M.; Tsonopoulos, C.; Steele, W. E.; J. Chem. Thermodyn. 1990, 22, 1075

32. Sabbah, R.; Bull. Soc. Chim. Fr. 1991, 128, 350.

33. Chickos, J. S.; Acree Jr., W. E.; J. Phys. Chem. Ref. Data 2002, 31, 537.

34. Sabbah, R.; El Watik, L.; Thermochim. Acta 1992, 197, 381.

35. Steele, W. V.; Chirico, R. D.; Cowell, A. B.; Nguyen, A.; Knipmeyer, S. E.; J. Chem. Thermodyn. 1995, 27, 1407.

36. Domalski, E. S. Em Experimental Chemical Thermodynamics, vol.1, Sunner, S.; Månson, M.; eds.; Pergamon Press: Oxford, 1979, cap.18.

37. Hubbard, W. N.; Scott, D. W.; Waddington, G.; Em Experimental Thermochemistry, vol. I, Rossini, F. D., ed.; Interscience: New York, 1956, cap. 5.

38. Gundry, H. A.; Harrop, D.; Head, A. J.; Lewis, G. B.; J. Chem. Thermodyn. 1969, $1,321$.

39. Bickerton, J.; Pilcher, G.; Al-Takhin, G.; J. Chem. Thermodyn. 1984, 16 , 373.

40. Ribeiro da Silva, M. D. M. C.; Santos, L. M. N. B. F.; Silva, A .L. R.; Fernandes, O.; Acree Jr., W. E.; J. Chem. Thermodyn. 2003, 35, 1093.

41. Ribeiro da Silva, M. A. V.; Ferrão, M. L. C. C. H.; Jiye, F.; J. Chem. Thermodyn. 1994, 26, 839.

42. Calvet, E.; Em Experimental Thermochemistry, vol. 1, Rossini F. D., ed.: Interscience Publishers, Inc: New York, 1956, cap. 12.

43. Calvet, E.; Em Experimental Thermochemistry, vol. 2, Skinner H. A., ed.; Interscience Publishers, Inc: New York, 1962, cap. 17.

44. Cox, J. D.; Pilcher, G.; Thermochemistry of Organic and Organometallic Compounds, Academic Press: London, 1970.

45. Adedeji, F. A.; Brown, D. L. S.; Connor, J. A.; Leung, M.; Paz-Andrade, M. I.; Skinner H. A.; J. Organomet. Chem. 1975, 97, 221.

46. Ribeiro da Silva, M. A. V.; Matos, M. A. R.; Amaral, L. M. P. F.; J. Chem. Thermodyn. 1995, 27, 565.

47. Santos, L. M. N. B. F.; Schroder, B.; Fernandes, O. O. P.; Ribeiro da Silva, M. A. V.; Thermochim. Acta 2004, 415, 15.

48. Monte, M. J. S.; Tese de Doutoramento, Faculdade de Ciências da Universidade do Porto, 1990.

49. Knudsen, M.; Ann. Phys. 1909, 28, 75.

50. Knudsen, M.; Ann. Phys. 1909, 28, 999.

51. Knudsen, M.; Ann. Phys. 1909, 29, 179.

52. Ribeiro da Silva, M. A. V.; Monte, M. J. S.; Santos, L. M. N. B. F.; J. Chem. Thermodyn. 2006, 38, 778.

53. Baboul, A. G.; Curtiss, L. A.; Redfern, P. C.; Raghavachari, K.; J. Chem. Phys. 1999, 11, 7650.

54. Becke, A. D.; Phys. Rev. A 1988, 38, 3098.

55. Lee, C.; Yang, W.; Parr, R. G.; Phys. Rev. B 1988, 37, 785.

56. Jensen, F.; Introduction to Computational Chemistry, John Wiley \& Sons, Ltd.: Chichester, West Sussex, England, 2007.

57. Merrick, J. P.; Moran, D.; Radom, L.; J. Phys. Chem. 2007, 111, 11683.

58. Pople, J. A.; Head-Gordon, M.; Raghavachari, K.; J. Chem. Phys. 1987, $87,5968$.

59. Møller, C.; Plesset, M. S.; Phys. Rev. 1934, 46, 618.

60. Hehre, W. J.; Ditchfield, R.; Radom, L.; Pople, J. A.; J. Am. Chem. Soc. 1970, 92, 4796.

61. Radom, L.; Hehre, W. J.; Pople, J. A.; J. Am. Chem. Soc. 1971, 93, 289.

62. George, P.; Trachtman, M.; Bock, C. W.; Brett, A. M.; Tetrahedron 1976, 32,317 .

63. George, P.; Trachtman, M.; Bock, C. W.; Brett, A. M.; J. Chem. Soc. Perkin Trans. 1976, 2, 1222.

64. Freitas, V. L. S., Tese de Doutoramento, Faculdade de Ciências da Universidade do Porto, 2011.

65. Freitas, V. L. S.; Gomes, J. R. B.; Ribeiro da Silva, M. D. M. C.; J. Chem. Thermodyn. 2009, 41, 1199. 
66. Freitas, V. L. S.; Gomes, J. R. B.; Ribeiro da Silva, M. D. M. C.; J. Chem. Thermodyn. 2010, 42, 1248.

67. Freitas, V. L. S.; Gomes, J. R. B.; Ribeiro da Silva, M. D. M. C.; Struct. Chem. 2013, 24, 661.

68. Freitas, V. L. S.; Monte, M. J. S.; Santos, L. M. N. B. F.; Gomes, J. R. B.; Ribeiro da Silva, M. D. M. C.; J. Phys. Chem. A 2009, 113, 12988.

69. Freitas, V. L. S.; Ribeiro da Silva, M. D. M. C.; Gomes, J. R. B.; J. Mol. Struct.: Theochem 2010, 946, 20.

70. Freitas, V. L. S.; Gomes, J. R. B.; Ribeiro da Silva, M. D. M. C.; J. Chem. Thermodyn. 2009, 42, 251.
71. Freitas, V. L. S.; Gomes, J. R. B.; Ribeiro da Silva, M. D. M. C.; J. Therm. Anal. Calorim. 2009, 97, 827.

72 Freitas, V. L. S.; Gomes, J. R. B.; Gales, L.; Damas, A. M.; Ribeiro da Silva, M. D. M. C.; J. Chem. Eng. Data 2010, 55, 5009.

73. Freitas, V. L. S.; Gomes, J. R. B.; Ribeiro da Silva, M. D. M. C.; J. Chem. Thermodyn. 2012, 54, 108.

74. Steele, W. V.; Chirico, R. D.; Cooperative Agreement No. FC22$83 F E 60149$ (NIPEP-457), IIT Research Institute, NIPEP, Bartlesville, OK 74005, 1990, 1. 


\section{EFEITOS ENERGÉTICO-ESTRUTURAIS EM COMPOSTOS HETEROPOLICÍCLICOS COM OXIGÉNIO OU ENXOFRE}

Vera L. S. Freitas e Maria D. M. C. Ribeiro da Silva*

Centro de Investigação em Química, Departamento de Química e Bioquímica, Faculdade de Ciências, Universidade do Porto, Rua do Campo Alegre, 687, P-4169-007 Porto, Portugal

José R. B. Gomes

CICECO, Departamento de Química, Universidade de Aveiro, Campus Universitário de Santiago, P-3810-193 Aveiro, Portugal

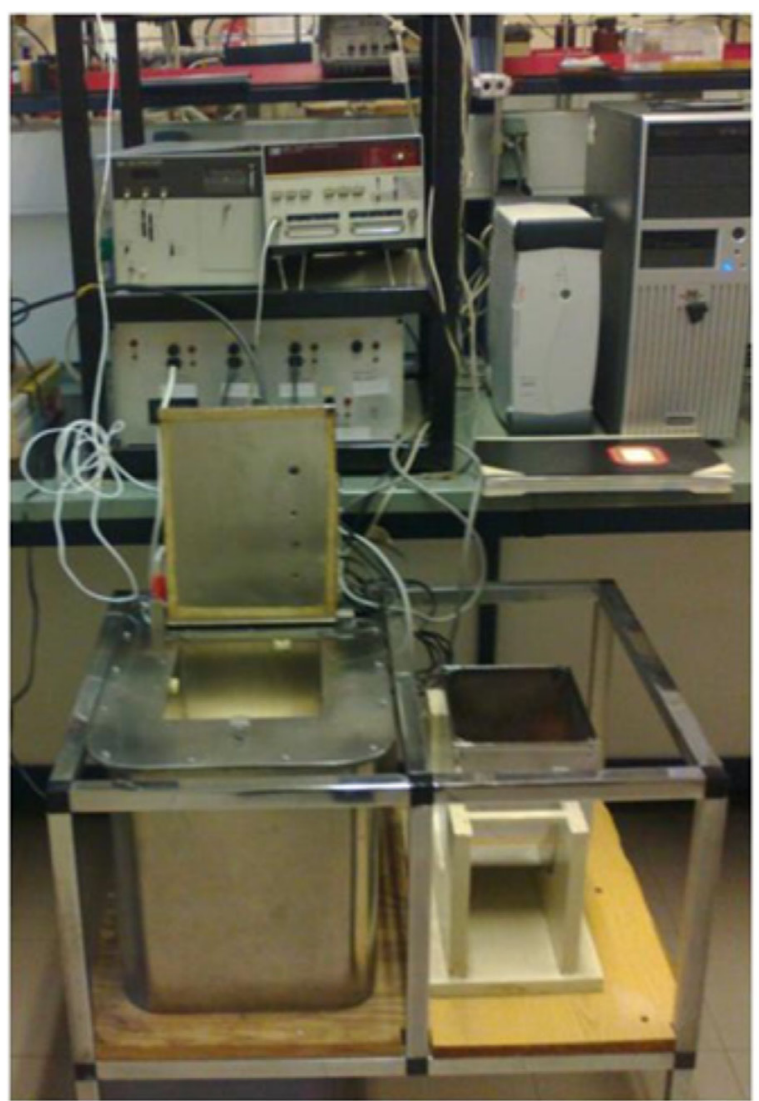

Vista geral do sistema de calorimetria de combustão em bomba rotativa.

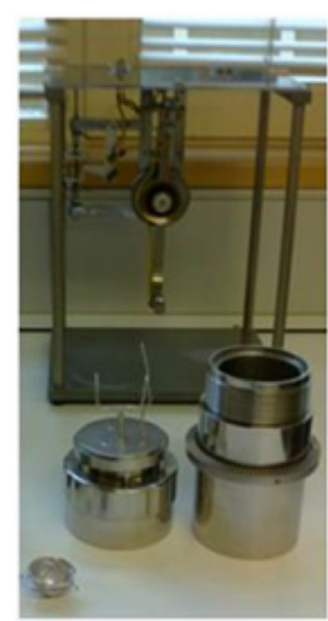

(atrás) Suporte da bomba rotativa; (à frente da esquerda para a direita) Cadinho de platina, cabeça da bomba e corpo da bomba.

Figura 1S. Sistema de calorimetria de combustão em bomba rotativa ${ }^{1}$ 


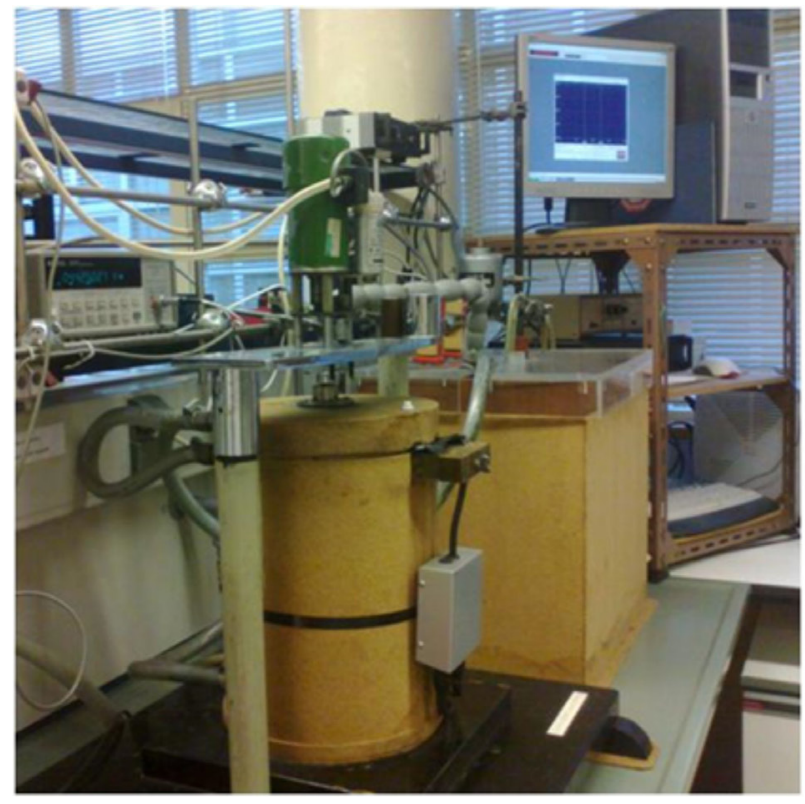

Vista geral do sistema de calorimetria de combustão em bomba estática.

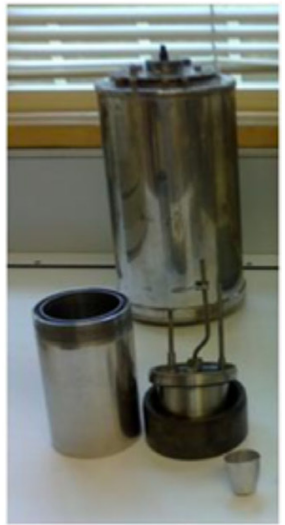

(atrás) Vaso calorimétrico; (à frente da esquerda para a direita) corpo da bomba, cabeça da bomba e cadinho de platina.

Figura 2S. Sistema de calorimetria de combustão em bomba estática ${ }^{2}$

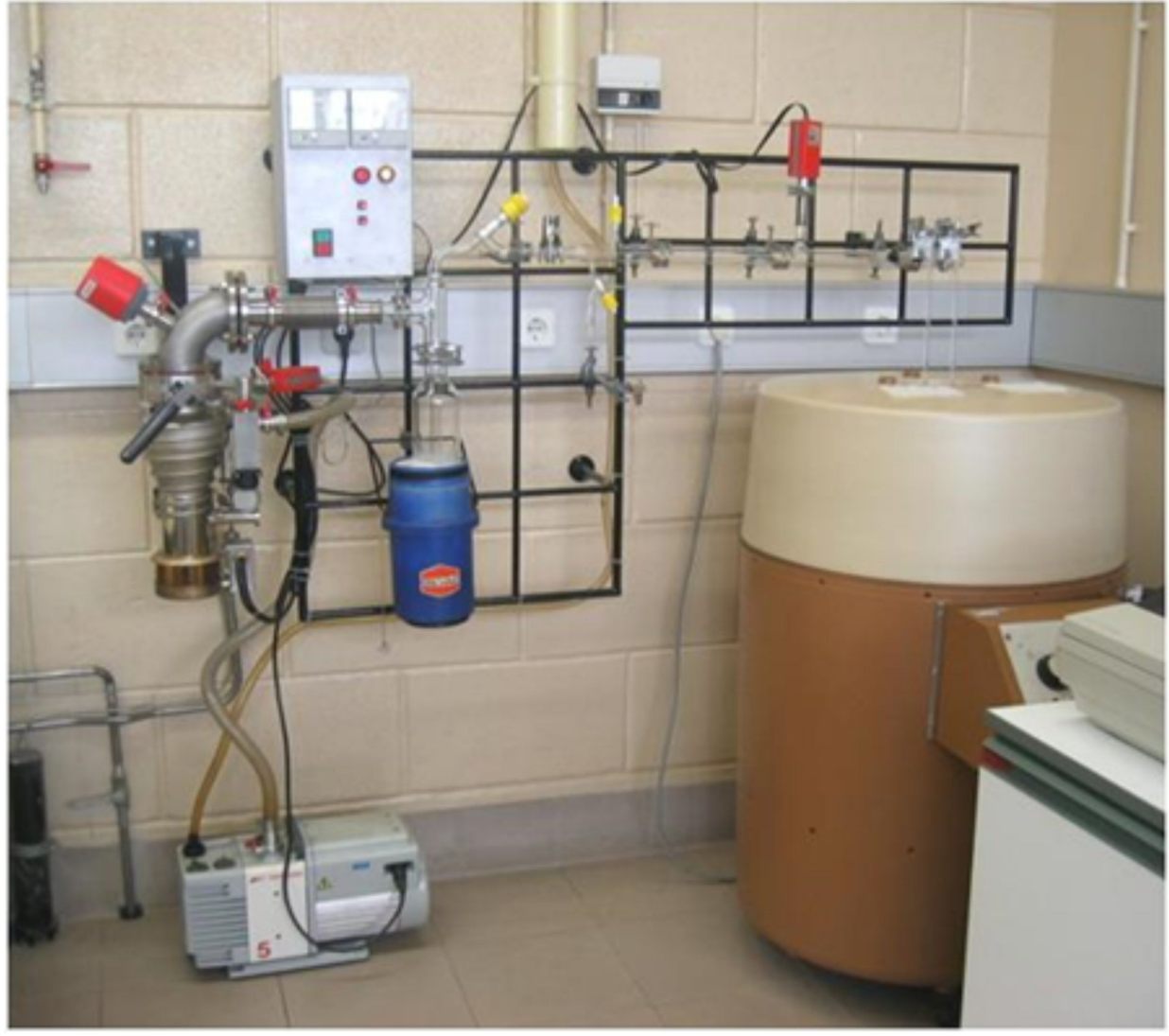

Figura 3S. Sistema de microcalorimetria Calvet ${ }^{3}$ 


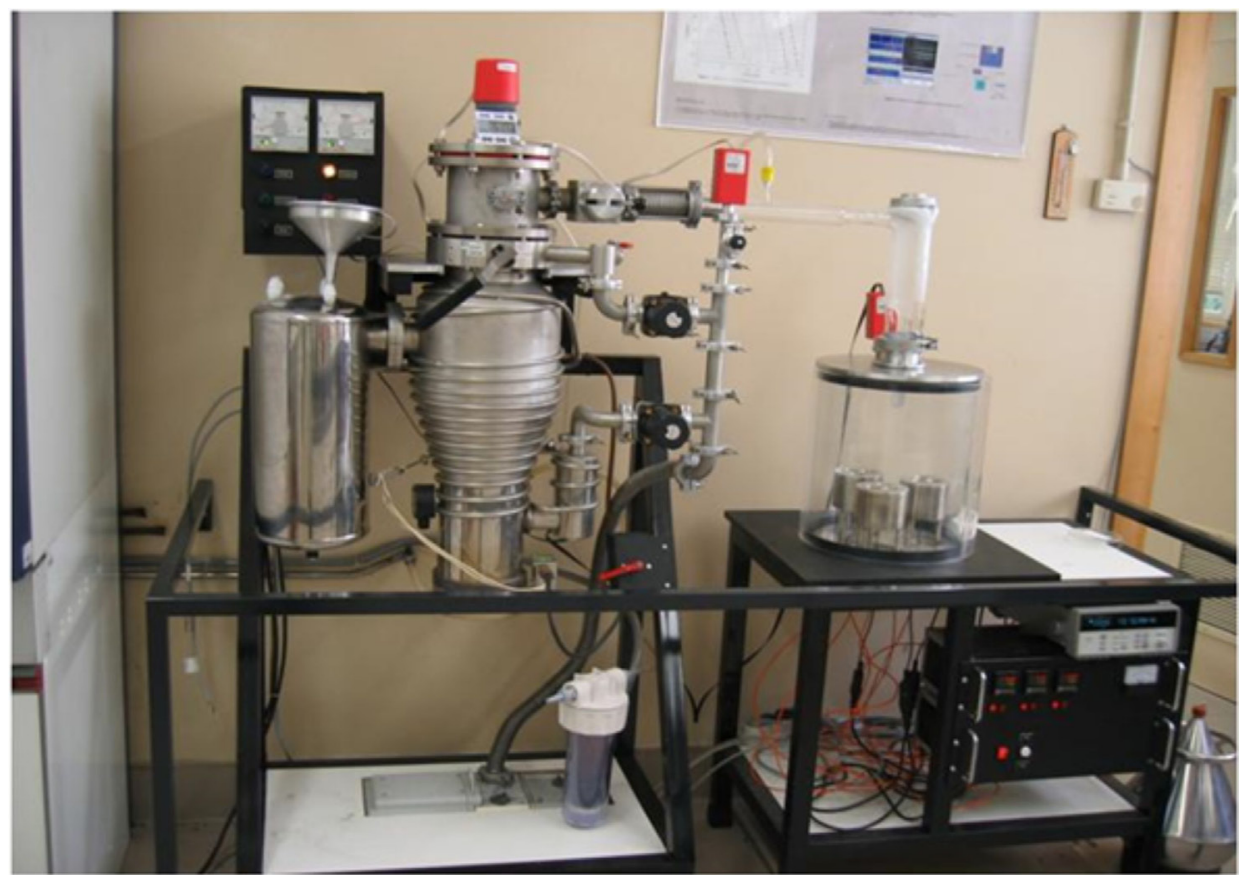

Figura 4S. Sistema de efusão de Knudsen ${ }^{4}$

\section{REFERÊNCIAS}

1. Ribeiro da Silva, M. A. V.; Ferrão, M. L. C. C. H.; Jiye, F.; J. Chem. Thermodyn. 1994, 26, 839.

2. Ribeiro da Silva, M. D. M. C.; Santos, L. M. N. B. F.; Silva, A .L. R.; Fernandes, O.; Acree Jr., W. E.; J. Chem. Thermodyn. $2003,35,1093$.

3. Santos, L. M. N. B. F.; Schroder, B.; Fernandes, O. O. P.; Ribeiro da Silva, M. A. V.; Thermochim. Acta 2004, 415, 15.

4. Ribeiro da Silva, M. A. V.; Monte, M. J. S.; Santos, L. M. N. B. F.; J. Chem. Thermodyn. 2006, 38, 778. 